\title{
Serum Anjiogenesis Factors of Neuropilin-1 and Neuropilin-2 Levels in Clinically Isolated Syndrome and Relapsing-Remitting Multiple Sclerosis Patients
}

Ahmet Dönder ( $\square$ ahmetdundar83@hotmail.com )

Mardin Artuklu Universitesi

Hasan Hüseyin ÖZDEMiR

United Hospital

Hamza ASLANHAN

Dicle Universitesi

Research article

Keywords: Neuropilin -1, Neuropilin -2, RRMS, CIS, Angiogenesis

Posted Date: September 14th, 2020

DOl: https://doi.org/10.21203/rs.3.rs-67589/v1

License: (c) (i) This work is licensed under a Creative Commons Attribution 4.0 International License.

Read Full License 


\section{Abstract}

Background: Several of the molecular constituent factors in the pathophysiology of Multiple Sclerosis (MS). Neuropilins are transmembrane glycoproteins which have to be receptors for the vascular endothelial growth factor (VEGF) family of angiogenesis factors. The role of angiogenesis factors of Neuropilin-1 and 2 in the pathology of MS is unknown.

Methods: We aimed to investigate levels of serum Neuropilin-1 and 2 in Relapsing-Remitting Multiple Sclerosis (RRMS), and Clinically Isolated Syndrome Patients (CIS) and to investigate a correlation with, age, sex, Expanded Disability Status Scale (EDSS) and relationship with immunomodulatory therapy. Serum Neuropilin-1 and 2 concentrations of 46 RRMS patients and 28 CIS patients and 45 healthy control group were analyzed. Clinical status was evaulated using the Expanded Disability Status Scale (EDSS).

Results: Neuropilin-1 and 2 concentrations matched for immumodulatuar treatment, age and sex at a group level.Neuropilin levels were found to be significantly higher in the CIS and RRMS patient's groups compared with the control group ( $\mathrm{p} \otimes 0,001)$. No statistically significant difference was found between groups; age, immunmodulatuar treatment, EDSS and gender.

Conclusions: Neuropilin-1 and 2 levels have been shown to increase in RRMS and CIS patients. Neuropilins, one of the factors of angiogenesis, may be effective in pathophysiology since the first period of the disease.

\section{Background}

Multiple Scleorosis (MS) is a chronic inflammatory disease of characterized by demyelination of the central nervous system (CNS). Clinically isolated syndrome (CIS) refers to a single clinical attack of CNS inflammatory demyelinating symptoms that are lead to MS. One of the most prominent clinical features of MS is relapsing remitting RRMS. Several factors play an important role in the MS pathophysiology [1]. Abnormalities in blood vessels have been observed along with impaired barrier function seen in MS lesions [2]. Blood-brain barrier (BBB) deficiency has also been documented as an altered expression of endothelial tight junction proteins, changes of vascular basement membrane molecules and pericytes in MS types [3-6].

The increased BBB permeability driven by the release of Vascular Endothelial Growth Factor (VEGF), that is also regulates vessel chemotactic and growth for lymphocytes and monocytes, promoting neuroinflammation [7]. The studies suggested that angiogenesis, the formation of new vessels, is detrimental to MS pathogenesis [8, 9]. Angiogenesis is found in MS demyelinating lesions following VEGF release and the production of several other angiogenic molecules. The Neuropilins are a family of essential cell surface receptors involved in multiple main cellular signaling cascades. They are expressed on many cells of the immune system, including dendritic cells, macrophages, mast cells, and basophils [10]. Neuropiline (NRP) 2 is known as the homolog of neuropiline-1. Neuropiline (NRP) 1 and Neuropiline (NRP) -2, which play a role in neuronal guidance and angiogenesis, function as both class 3 semaphorins 
(SEMA) and VEGF coreceptors. NRP molecules have been classified as regulators in nervous system development due to their ability to act as coreceptors with plexin, a specially secreted member of the semaphorin family that has neuronal guidance functions [11-14]. The potential involvement of NRP-1 in the pathogenesis of neurological diseases had been indicated by evidence showing that NRP1 protein levels are induced in the experimental cerebral ischemic neurons models [15]. Also, NRP-1 has been implicated to play a role in the immunological synapse and the pathogenesis of experimental autoimmune encephalomyelitis (EAE) [16].

In light of the literature, NRP-1 levels were increased in microvascular endoytelial cells in multiple sclerosis lesions and in human neuro inflanmatory diseases [17]. The serum values of neuropilins and the relationship between CIS and RRMS are unknown. We first evaluated this relationship in the literature.

\section{Methods}

This study was conducted in the Neurology Department of Dicle University, Diyarbakir, Turkey. A 46 patients with definite clinical RRMS, 28 CIS patients and 45 healthy control subjects were enrolled [18]. Our study included RRMS and CIS patients coming to the neurology outpatient clinic of Dicle University School of Medicine. Disability was evaluated using the Expanded Disability Status Scale (EDSS) [19]. Our study was conducted after the approval of Dicle University Clinical Research Ethics Committee (2014/233). Informed consent forms were signed by the individuals in both the patient and control groups.

In this study, only patients receiving interferon $1 \mathrm{a}$ and interferon $1 \mathrm{~b}$ treatment were included in the RRMS group. Serum samples were collected before patients in the Cis group received immunotherapy. In the RRMS group disease duration was defined as the time in years between diagnosis and blood sampling. Hypertension, clinical infection, cancer, pregnancy, diabetes mellitus, individuals under 18 years of age, inflammatory diseases were determined as exclusion criteria in our study.

\section{Biochemical analysis of Serum Neuropilin-1 and Neuropilin-2}

Blood was collected from RRMS, CIS and control group. The collected blood was centrifuged for 10 minutes at $3000 \mathrm{rpm}$ in accordance with the manufacturer's protocol after 15 minutes of waiting for the laboratory. The received serums waited in the freezer at -80 centigtrat degrees until the working day. Serum Neuropilin-1 and Neuropilin-2 kits measured using commercially available enzyme-linked immunosorbent assay (ELISA) kits (eastbiopharm, Kit LTD, China). Neuropilin-1 and Neuropilin-2 serum samples were measured at $450 \mathrm{~nm}$ and recorded by Absorbance Microtiter Plate Reader (ELx800TM, BIOTEK instruments, USA).

\section{Statistical analysis}

The mean, standard deviation, Minimum and maximum values are expressed, while the descriptive statistics for continuous variables are expressed as numbers and percentages for categorical variables. 
In comparison with the Kruskal-Wallis test, a bonferroni correction was made to determine which group the statistical difference was due to, and a 2-point comparison was made with the Mann-Whitney $U$ test. Spearmankorelation analysis was used to determine the relationship between the data. The statistical significance level of the calculations was taken as $5 \%$ and the calculations were made with SPSS (Statistical Packagee for social sciences) version 18.0.

\section{Results}

The results of this study are summarized in Tables 1, 2 and 3. The mean age of the RRMS patients (31 females and 15 males), CIS patients ( 15 females and 13 males), and the control group ( 30 females and 15 males) was $30.3 \pm 9.25$ and $30.28 \pm 9.13$ years, respectively. The mean duration of disease and EDSS was $4.79 \pm 4.0$ years and $1.97 \pm 2,00$ respectively.

Neuropilin 1 serum levels were significantly increased in the CIS group $(2,51 \mathrm{pg} / \mathrm{mL}(1,63-29,86)$ and RRMS $(2,40 \mathrm{pg} / \mathrm{mL}(1,27-32,30)$ group compared to healthy controls $(1,35 \mathrm{pg} / \mathrm{mL}(0,10-13,85)$ $(\mathrm{p} \bowtie 0,001)$.

Neuropilin 2 serum levels were significantly increased in the CIS group $(3,13 \mathrm{pg} / \mathrm{mL}(1,21-33,2)$ and RRMS $(3,28 \mathrm{pg} / \mathrm{mL}(0,98-30,74))$ group compared to healthy controls $(1,15 \mathrm{pg} / \mathrm{mL}(0,42-3,2)(\mathrm{p} \otimes 0,001)$.

Serum Neuropilin-1 and 2 levels were found to be higher in both RRMS patients and CIS patients when compared with controls. This difference was statistically significant compared with the control group $(\mathrm{p} \otimes 0,001)$.

A 25 of the RRMS patients were using interferon $\beta 1 a$ and 21 patients were using beta $1 \beta$. There is no difference between patient and treatment groups. Table-2.

There were no significant Neuropilin 1 and 2 levels differences between duration of disease and EDSS. Table-3.

Table 1

Neuropilin-1 and Neuropilin-2 levels in Multiple Sclerosis (MS) patients, CIS patients and healthy controls

\begin{tabular}{|c|c|c|c|c|c|}
\hline & & Control group [ $n=45]$ & Cis patients[n = 28] & RRMs patients [n = 46] & \\
\hline \multirow[t]{2}{*}{ Gender } & Female & 30 & 15 & 31 & \multirow[t]{2}{*}{$\mathrm{p}$} \\
\hline & Male & 15 & 13 & 15 & \\
\hline \multirow{2}{*}{\multicolumn{2}{|c|}{$\begin{array}{l}\text { Neuropilin-1 } \\
\text { [pg/mL] }\end{array}$}} & 1,35 & 2,51 ** & $2,40 * \star$ & \multirow[t]{2}{*}{$\varangle 0,001$} \\
\hline & & {$[0,10-13,85]$} & {$[1,63-29,86]$} & {$[1,27-32,30]$} & \\
\hline \multirow{2}{*}{\multicolumn{2}{|c|}{$\begin{array}{l}\text { Neuropilin-2 } \\
\text { [pg/mL] }\end{array}$}} & 1,15 & $3,13 * \star$ & $3,28 * \star$ & \multirow[t]{2}{*}{$\otimes 0,001$} \\
\hline & & {$[0,42-3,2]$} & {$[1,21-33,2]$} & {$[0,98-30,74]$} & \\
\hline
\end{tabular}


Notes: Data areexpressed as median[Min-Max]

$p<0.001$, comparison withcontrolgroup; **the degree of significance of comparison between CIS group and MS groups.

Table 2

Neuropilin-1 and Neuropilin-2 levels in Multiple Sclerosis [MS] patients, n CIS patients and healthy controls

\begin{tabular}{|c|c|c|c|c|c|}
\hline & & $\begin{array}{l}\text { Control group } \\
{[n=45]}\end{array}$ & $\begin{array}{l}\text { interferon1a } \\
{[n=21]}\end{array}$ & $\begin{array}{l}\text { İnterferon1 } \beta \\
{[n=25]}\end{array}$ & \\
\hline \multirow[t]{2}{*}{ Gender } & Female & 30 & 14 & 14 & \multirow[t]{2}{*}{$\mathrm{p}$} \\
\hline & Male & 15 & 7 & 11 & \\
\hline \multirow{2}{*}{\multicolumn{2}{|c|}{$\begin{array}{l}\text { Neuropilin-1 } \\
{[\mathrm{pg} / \mathrm{mL}]}\end{array}$}} & 1,35 & $2,41 * \star$ & $2,40 * \star$ & \multirow[t]{2}{*}{$\nabla 0,001$} \\
\hline & & {$[0,10-13,85]$} & {$[1,68-32,30]$} & {$[1,27-19,90]$} & \\
\hline \multirow{2}{*}{\multicolumn{2}{|c|}{$\begin{array}{l}\text { Neuropilin-2 } \\
{[\mathrm{pg} / \mathrm{mL}]}\end{array}$}} & 1,15 & $3,78 * \star$ & $3,13 * \star$ & \multirow[t]{2}{*}{$\otimes 0,001$} \\
\hline & & {$[0,42-3,2]$} & {$[0,98-30,74]$} & {$[1,65-13,86]$} & \\
\hline
\end{tabular}

Notes: Data are expressed as median[Min-Max]

$<0.001$, comparison with Control group; **thedegree of significance of comparison between CIS group and MS groups.

Table 3

Correlation coefficients between parameters in MS group

\begin{tabular}{|c|c|c|c|c|c|c|}
\hline & & Year & Disease duration & EDSS & Neuropilin-1 & Neuropilin-2 \\
\hline \multirow[t]{2}{*}{ Year } & $r$ & 1.000 & & & & \\
\hline & $p$ & & & & & \\
\hline \multirow[t]{2}{*}{ Diseasae duration } & $r$ & 371 & 1.000 & & & \\
\hline & $p$ & 011 & & & & \\
\hline \multirow[t]{2}{*}{ EDSS } & $r$ & 208 & ,243 & 1.000 & & \\
\hline & $\mathrm{p}$ & 165 & 104 & & & \\
\hline \multirow[t]{2}{*}{ Neuropilin-1 } & $r$ & ,049 & ,016 & 149 & 1.000 & \\
\hline & $\mathrm{p}$ & ,598 & ,916 & ,324 & & \\
\hline \multirow[t]{2}{*}{ Neuropilin-2 } & $r$ & ,047 &,- 119 & ,267 &, $535^{\star \star}$ & 1.000 \\
\hline & $\mathrm{p}$ & 614 & ,431 & ,073 & ,000 & \\
\hline
\end{tabular}

$*: p<0.05 ; * *: p<0.01$ 


\section{Discussion}

In the present study, we showed Neuropilin-1 and 2 are elevated in serum from CIS and RRMS compared with healthy controls. We report no correlation between age, EDSS, disease duration and treatments group at RRMS patients. This is the first study to evaluate of relationship between CIS, RRMS and serum Neuropilin 1 and 2.

Clinically Isolated Syndrome is often the first manifestation of MS. The majority of patients with CIS will go on to develop MS. Studies evaluating the effect of angiogenesis in these patients are limited. Boroujerdi et al.stated that this increase in pre-symphomatic increase in angiogenesis supports pathogenic relay in the pathogenesis of MS [20]. In our study, high levels in CIS patients support this view. These results may indicate that angiogenesis may occur even in the early stages of the disease.

RRMS is the most common form of MS disease, and the disease progresses over time. Multiple sclerosis patient serum and CNS paranchyma proinflammatory cytokines were elevated, as well as the progression of MS and proinflammatory cytokines were reported in Korea. Ribatti et al. demonstrated that angiogenic activity Cerebrospinal fluid samples from MS patients and confirm the importance of angiogenesis as a key event in MS pathogenesis and progression [21]. RRMS patients had significantly higher neuropiline levels, but we could not find a correlation with disease duration. MacMillan et al. showed that pharmacological inhibition of angiogenesis with various compounds suggests that it is beneficial for disease outcome in the EAE model [22]. Interestingly, Neuropiline values were similiar between RRMS and CIS group. Also, no correlations between RRMS and EDSS. This may be related to drug therapy. On the other hand, there was no difference between the interferon groups. Drugs with this similar mechanism of MS pathogenesis show that they may affects angiogenesis similarly.

In summary, we first showed that Neuropilins serum levels are increased in RRMS and CIS patients. We suggest that Neuropilins may play a role in MS pathogenesis especially from the beginning of the disease. Further studies should be undertaken to further test the possible role of Neuropilins in MS pathogenesis.

\section{Abbreviations}

MS: Multiple Sclerosis; VEGF: Vascular endothelial growth factor, RRMS :Relapsing-Remitting Multiple Sclerosis; CIS: Clinically Isolated Syndrome Patients; EDSS :Expanded Disability Status Scale; CNS:

Central nervous system; NRP: Neuropiline; BBB: Blood-brain barrier, SEMA: Semaphorin; EAE:

Encephalomyelitis; SPSS:Statistical Packagee for social sciences

\section{Declarations}

Acknowledgements

Not applicable. 
Conflict of interest: The authors declare that there is no confict of interest.

Ethics approval and consent to participate: The manuscript was approved by the Dicle University Clinical Research Ethics Committee (2014/233).

Consent for publication

Not applicable.

Competing interests

The authors declare that they have no competing interests.

Funding

Research was supported by the Research Fund of the Dicle University.

Availability of data and materials

All data are available without restriction from corresponding author on reasonable request.

Ethics approval and consent to participate

The project was approved through the Ethics Committee of Dicle University

(Ethics number: 2014-233)

Authors' contributions $A D$ and HHÖ participated in study design. $A D$ and HA participated in statistical analysis. All authors read and approved the final manuscript.

\section{References}

1. Keegan BM. Noseworthy JH Multiple sclerosis. Annu Rev Med. 2002;53:285-302.

2. Lengfeld J, Cutforth T, Agalliu D. The role of angiogenesis in the pathology of multiple sclerosis. Vasc Cell. 2014;6(1):23.

3. Alvarez Jl, Cayrol R, Prat A. Disruption of central nervous system barriers in multiple sclerosis. Biochim Biophys Acta. 1812(2):252-64.

4. Claudio L, Raine CS, Brosnan CF. Evidence of persistent blood-brain barrier abnormalities in chronicprogressive multiple sclerosis. Acta Neuropathol. 1995;90(3):228-38.

5. Kirk J, Plumb J, Mirakhur M, McQuaid S. Tight junctional abnormality in multiple sclerosis white matter affects all calibers of vessel and is associated with blood-brain barrier leakage and active demyelination. J Pathol. 2003;201(2):319-27.

6. McQuaid S, Kirk JT. The blood-brain barrier in multiple sclerosis. Int Congress Series. 2005; 1277:235-243. 
7. Girolamo F, Coppola C, Ribatti D, Trojano M. Angiogenesis in multiple sclerosis and experimental autoimmune encephalomyelitis. Acta Neuropathol Commun. 2014; 22;2:84.

8. Kirk SL, Karlik SJ. VEGF and vascular changes in chronic neuroinflammation. J Autoimmun. 2003;21(4):353-63

9. Kirk S, Frank JA, Karlik S. Angiogenesis in multiple sclerosis: is it good, bad or an epiphenomenon? J Neurol Sci. 2004; 15;217(2):125-30.

10. Immormino RM, Lauzier DC, Nakano H,HernandezM L, Alexis NE, Ghio AJ et al. Neuropilin-2 regulates airway inflammatory responses to inhaled lipopolysaccharide. Am J Physiol Lung Cell Mol Physiol. 2018;315(2):L202-L211.

11. Giger RJ, Cloutier JF, Sahay A, Prinjha RK, Levengood DV, Moore SE, et al. Neuropilin-2 is required in vivo for selective axon guidance responses to secreted semaphorins. Neuron 2000;25(1):29-41

12. Kolodkin AL, Ginty DD. Steering clear of semaphorins neuropilins sound the retreat. Neuron. 1997;19(6):1159-62.

13. He Z, Tessier-Lavigne M. Neuropilin is a receptor for the axonal chemorepellent Semaphorin III. Cell 1997; 22;90(4):739-51.

14. Elpek GÖ. Neuropilins and liver. World J Gastroenterol. 2015;21(23):7065-7073.

15. Zhang ZG, Tsang W, Zhang L, Powers C, Chopp M. Upregulation of neuropilin-1 in neovasculature after focal cerebral ischemia in the adult rat. J. Cereb. Blood Flow Metab. 2001;21(5):541-9

16. Solomon BD, Mueller C, Chae WJ, Alabanza LM, Bynoe MS. Neuropilin-1 attenuates autoreactivity in experimental autoimmune encephalomyelitis. Proc Natl Acad Sci U S A. 2011;108(5):2040-2045.

17. Wang Y, Cao Y, Mangalam AK, Guo Y , LaFrance-Corey R G, GamezJ D et al. Neuropilin-1 modulates interferon-Y-stimulated signaling in brain microvascular endothelial cells. J Cell Sci. 2016;129(20):3911-3921.

18. Polman $\mathrm{CH}$, Reingold SC, Banwell B, Clanet M, Cohen JA, Filippi M, et al. Diagnostic criteria for multiple sclerosis: 2010 revisions to the McDonald criteria. Ann Neurol 2011; 69:292-302.

19. Kurtzke, J.F. Rating neurologic impairment in multiple sclerosis: An expanded disability status scale (EDSS). Neurology $1983 ; 33(11): 1444-52$

20. Boroujerdi A, Welser-Alves JV, Milner R. Extensive vascular remodeling in the spinal cord of presymptomatic experimental autoimmune encephalomyelitis mice; increased vessel expression of fibronectin and the alpha5beta1 integrin. Exp Neurol. 2013, 250:43-51.

21. Ribatti $D$, laffaldano $P$, Marinaccio $C$, Trojano $M$. First evidence of in vivo pro-angiogenic activity of cerebrospinal fluid samples from multiple sclerosis patients. Clin Exp Med. 2016;16(1):103-107.

22. MacMillan CJ, Doucette CD, Warford J, Furlong SJ, Hoskin DW, Easton AS: Murine experimental autoimmune encephalomyelitis is diminished by treatment with the angiogenesis inhibitors B204.1.1 and angiostatin (K1-3). PLoS One $2014 ; 26 ; 9(2)$ :e89770

\section{Supplementary Files}


This is a list of supplementary files associated with this preprint. Click to download.

- SerumAnjiogenesisFactorsofNeuropilin12titlepage.docx 